\title{
New Approach to Dynamic and Seamless Management of Network and Computational Resources in a Heterogeneous Grid Environment
}

\author{
Bartosz Belter, Artur Binczewski, Miłosz Przywecki, Maciej Stroiński \\ Poznań Supercomputing and Networking Center, Z. Noskowskiego 12/14, 61-704 Poznań, Poland \\ \{bart/artur/mprzyw/stroins\}@man.poznan.pl
}

(Received: 25 August 2010; revised: 29 October 2010; published online: 23 November 2010))

\begin{abstract}
The advent of network innovations in optical networks has a significant influence on computational infrastructures in research projects. Grid applications and middleware, not yet aware of underlying network and its services, have been recently equipped with powerful tools and plug-ins to control and manage not only the computational, but also network infrastructures. However, at this point of time, Grid middleware, even if aware of network services, is not capable of seamless control and management of both, Grid and network resources. The goal of this paper is to introduce a novel approach to dynamic and on-demand management of Grid and network resources in the PHOSPHORUS project.
\end{abstract}

Key words: Grid infrastructure, optical networks, network management, network control plane, GMPLS, NRPS

\section{INTRODUCTION}

Progress in the field of distributed computing and development of advanced Grid services has a significant influence on the concrete results of cooperation between distributed computing centers in various parts of the world. Advanced applications using remote access to distributed computing resources, data repositories and laboratories impose strong requirements on data networks. Very often these requirements can be met only by technologically advanced optical networks. Guaranteed availability of bandwidth for data transfer between computing nodes, low and stable over time and the minimum packet loss are just some of the parameters that are important for the new generation networks. Moreover, the problem of scalability, particularly in the context of an increase of the size of Grid systems with high requirements to the network, significantly affects the complexity of infrastructure management systems, mainly due to the heterogeneity of the environment and the potential administrative divisions.

In order to increase processing efficiency of distributed computing, it is necessary to automate processes related to the management of infrastructure components. This is particularly important in the context of Grid environments connected via optical networks. Depending on the type of computing application, it is a network layer which is responsible for allocating inter-connections between computing sites with particular requirements on duration and the requested bandwidth. The possibility of a dynamic allocation of connections between computing sites is an essential element which allows for the efficient and effective use of optical networks in complex computational processes.

The PHOSPHORUS project addresses the issue of delivering advanced network services to Grid users and applications interconnected by high speed optical network infrastructure. The goal of the project is to make the Grid applications aware of the underlying network and to make the network infrastructure aware of available Grid resources. The project also enhances solutions already available in some networks by integrating existing Network Resource Provisioning Systems (NRPS) via scalable and open HARMONY component [30]. New approach towards the seamless application-to-network integration has been taken by extending the GMPLS protocol stack with the Grid-related attributes to make the network control plane aware of capabilities of Grid-resources available in the network. 
This paper focuses on the PHOSPHORUS approach to seamless management of the network and computational resources. The paper is organized as follows: Chapter II gives an overview of the related work in other research projects, Chapters III and IV describe the proposed PHOSPHORUS architectural model, with particular focus on the network/Grid management components, respectively. Chapter V discusses in detail the two integral parts of the PHOSPHORUS architecture, the $\mathrm{G}^{2}$ MPLS protocol stack and HARMONY, the multi-domain provisioning system.

\section{RELATED WORK}

Over the last few years, a number of projects have addressed the issue of resource management in next generation networks [28]. Most of them, however, have narrowed down to the management of network infrastructure only. On the other hand, there is a great choice of Grid management platforms to coordinate the allocation of computational resources. In consequence, at this point of time there is a great number of available solutions dealing with management of the network resources, while there is almost nothing on the market to provide seamless management of both, network and Grid resources.

This chapter will take a deeper look at the solutions available as a result of research projects in Europe, the United States and Japan. The first sub-section considers possible solutions to control and manage the networking infrastructure and offering it as a service to Grid applications and middleware. The second sub-section goes into details of the existing Grid middleware solutions and computational applications.

\section{II.1. Network Control and Management Planes}

The following section describes the existing solutions for the management of the networking infrastructures. All solutions presented in subsequent sections expose the infrastructure and its services for use by advanced scientific applications, Grid brokers and schedulers.

\section{II.1.1. ARGON: Allocation and Reservation in Grid-enabled Optic Networks}

ARGON (Allocation and Reservation in Grid-enabled Optic Networks) [4] is a component which was developed to manage resources of the advanced network equipment as it is present in the testbed created in the VIOLA project.

The project, which was funded by the German Ministry for Education and Research, ended in April 2007. ARGON was designed to provide a set of network related services to the Grid community, e.g. advance reservations can be requested by the upper layer (e.g. Grid Meta Schedulers or Grid applications). This includes the setup of network connections if the requested resources are available for the specified amount of time. At this level, ARGON hides the details of the network technologies, i.e. the user or application specifies the Quality of Service (QoS) requirements for a service and describes the service endpoints. ARGON maps abstract premium connectivity services to specific layer 2 and layer 3 network services via Multiprotocol Label Switching (MPLS) as well as point-to-point connectivity services via Generalized Multiprotocol Label Switching (GMPLS).

ARGON integrates with UNICORE middleware [24] using its Meta Scheduler Service (MSS) [6] element. This service is a core module which allows integration with Grid applications. An important functionality of MSS is its ability to coordinate allocation of multiple resources in multiple sites (domains) thus providing inter-working capabilities between different domains. To achieve this functionality, MSS implements adapters which provide communication between the Meta Scheduler Service and local Resource Management Systems.

All signaling protocols implemented by the service depend on the underlying hardware platforms. ARGON handles MPLS and GMPLS networks and L2VPN services (VLL and VPLS). It implements RSVP-TE (via proxies), SNMP protocol and Command Line Interface (CLI) interfaces and provides possibilities to implement other protocols as well. OSPF-TE is also used in the VIOLA testbed to transport data regarding available paths and links.

\section{II.1.2. DRAC: Dynamic Resource Allocation Controller}

The Dynamic Resource Allocation Controller (DRAC) is a commercial software developed by Nortel Networks and it allows to provide a function of the advance reservation of bandwidth [7]. The DRAC application is developed in close cooperation with SURFnet and the University of Amsterdam. The source code is available from Nortel Networks on a commercial license. The DRAC platform offers the single- and multi-domain support for different technologies: Ethernet with VLANs, DWDM and SDH/SONET.

\section{II.1.3. DRAGON: Dynamic Resource Allocation via GMPLS Optical Networks}

The Dynamic Resource Allocation via GMPLS Optical Networks (DRAGON) is the Bandwidth on Demand project funded by the USA National Science Foundation 
(NSF). The main project goal is to develop an infrastructure for inter-domain network resource management in heterogeneous environments through GMPLS signaling [8].

The project has defined the DRAGON architecture, which aims to leverage the emergence and maturing of optical network technologies to develop and demonstrate the power and flexibility of a "hybrid" packet and circuit switched network infrastructure.

DRAGON has been based on the Open Source GMPLS software to enable multi-domain, multi-layer, multi-service provisioning with robust levels of authentication, authorization, and accounting.

\section{II.1.4. G-Lambda}

The goal of G-Lambda is to establish a standard web services interface to network resource manager provided by network operators (Telecom operators) [11]. This interface may be used by application service providers (Grid resource managers/Grid brokers) or by end users to make the most of the available network operators service. The signaling is based on GMPLS, but the software does not support advance reservations.

The software has already been integrated with Grid facilities. For seamless integration of Grid and network management platforms the Grid Resource Scheduler (GRS) Web Service module has been implemented using Globus Toolkit 4 Java WS Core.

\section{II.1.5. AutoBAHN}

The AutoBAHN system aims at implementing an automatic bandwidth provisioning system for heterogeneous multi-domain NREN environments. Its objectives involve possible deployment of the Bandwidth on Demand (BoD) service over various network technologies, depending on NRENs requirements. The main focus of the research in AutoBAHN was focused on the most common technologies in European NRENs, in particular Ethernet and SDH. Network equipment can be managed directly by AutoBAHN, indirectly through vendor's NMS, or even with applications developed internally for particular NREN's needs.

The system has been developed as a pilot within the GN2 project co-funded by the European Commission as part of the Sixth Research and Development Framework Programme (FP6) [12].

\section{II.2. Grid management tools}

The following chapter presents the state-of-the-art in the area of Grid resource management and scheduling. Because the subject is wide and deeply investigated, only a few examples are considered, mainly these coming from the PHOSPHORUS project.

\section{II.2.1. UNICORE}

UNICORE (UNiform Interface to COmputing REsources) is a Grid computing technology that provides seamless, secure, and intuitive access to distributed Grid resources such as supercomputers or cluster systems and information stored in databases. UNICORE was developed in two projects funded by the German ministry for education and research (BMBF). In various European-funded projects, UNICORE has evolved to a full-grown and well-tested Grid middleware system over the years. UNICORE is used in daily production at several supercomputer centers worldwide. Beyond this production usage, UNICORE serves as a solid basis in many European and international research projects. The UNICORE technology is open source under the BSD licence and available at SourceForge, where new releases are published on a regular basis [13].

\section{II.2.2. GLOBUS Toolkit}

The GLOBUS Toolkit is an open source software toolkit used for building Grid systems and applications. The toolkit includes software for security, information infrastructure, resource management, data management, communication, fault detection, and portability. It is packaged as a set of components that can be used either independently or together to develop applications. The GLOBUS Toolkit was conceived to remove obstacles that prevent seamless collaboration. Its core services, interfaces and protocols allow users to access remote resources as if they were located within their own machine room while simultaneously preserving local control over who can use resources and when [14].

\section{II.2.3. GRIDGE}

The GRIDGE Toolkit is an open source software initiative aimed to help users to deploy ready-to-use Grid middleware services and create productive Grid infrastructures. All Gridge Toolkit software components have been integrated together and form a consistent distributed system following the same interface specification rules, license, quality assurance, and testing. Hence, Gridge is the Grid-In-The-Box solution that can be easily deployed on your infrastructure.

GRIDGE tools and services enable applications to take advantage of the dynamically changing Grid environment. These tools can deliver dynamic or utility computing to application users, developers and resource owners. Through supporting the shared, pooled and dynamically 
allocated resources and services managed by automated, policy-based Grid Resource Management System (GRMS), the GRIDGE offers the state-of-the-art dynamic Grid features to applications. The GRIDGE technology can be used by various kinds of businesses, including vendors, but also financial companies, service organizations, etc. [15].

\section{THE PHOSPHORUS ARCHITECTURE}

All abovementioned network and Grid solutions may easily address even the most demanding users' needs but mainly focus on solving specific Grid or networking problems separately and do not address the concept of seamless management of network and computational resources. The lack of a common management platform to orchestrate services provided independently by loosely coupled systems makes the overall management of technologically advanced Grid infrastructures difficult, if not possible at all. Trying to overcome the current limitations, the PHOSPHORUS project proposes a new architecture, where seamless provisioning of both Grid and network resources is the fundamental element of the architecture. Such integration enables scientific applications to use the infrastructure efficiently, simplifies access to resources, and shortens the time needed to setup scientific environments.

PHOSPHORUS proposes an innovative architecture which improves the performance of e-Science applications by ensuring single step straightforward procedures. These procedures can replace lengthy multiple steps of application setup, usually involving separate setup of network and Grid resources. To allow combined provisioning of both network and computational resources bridging elements have been identified and developed in the PHOSPHORUS project. The overall architecture of the proposed solution is presented in Fig. 1.

The overall architecture can be divided into five main parts. In the highest layer there are advanced e-Science applications which dynamically make use of different computational resources located in different sites, usually scattered geographically. The lowest layer contains all resources which are needed by the applications (e.g. computing power and storage) connected through a high speed optical network. Middle layers provide resource awareness to e-Science layer, and provide mechanisms for coordinated reservation of different resources from resources layer. To achieve this goal two approaches were implemented: extension of standard GMPLS control plane towards Grid Enabled GMPLS (G ${ }^{2}$ MPLS) and integration of different Network Resource Provisioning Systems

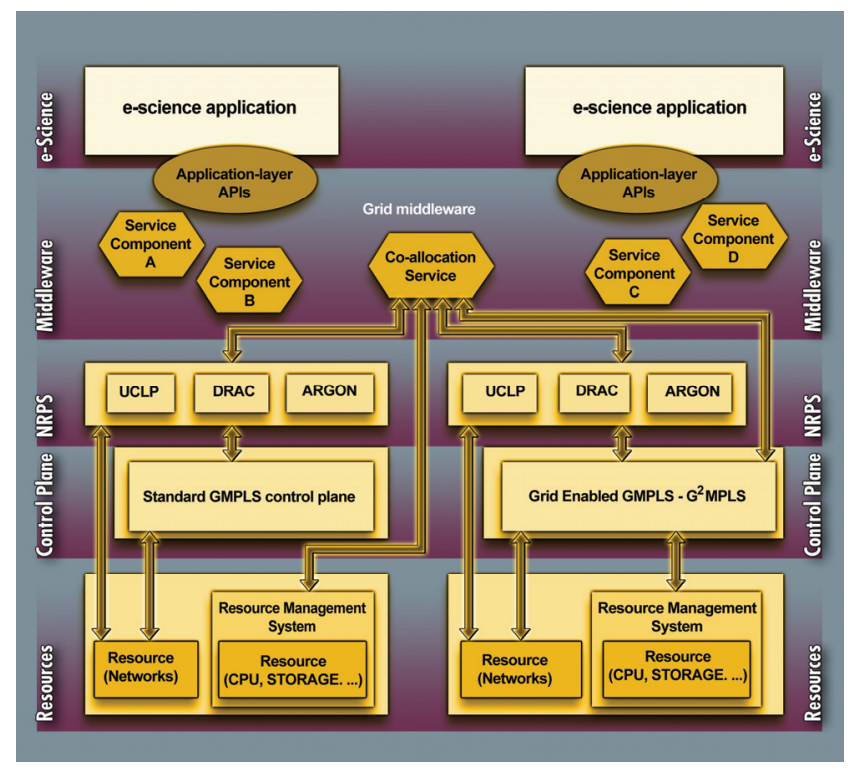

Fig. 1. Overall PHOSPHORUS architecture

(NRPS) to allow flexible reservations of the network in a multi-domain environment. In the first case, a standard GMPLS stack was extended to support the provisioning of network and Grid resources in a single step through a set of seamlessly integrated procedures. In the latter case, the development of the Network Service Plane (NSP) allowing interoperation of three NRPSes, namely User Controlled Lightpath (UCLP/ARGIA), Dynamic Resource Allocation Controller (DRAC) and Allocation and Reservation in Gridenabled Optical Networks (ARGON) was provided by the development of HARMONY - a multi-domain path provisioning system where users and Grid applications can book end-to-end paths and network resources. Interoperability of both solutions was provided to allow flexible use of these technologies in different parts of the network. The middleware layer, also located between applications and resources, is responsible for orchestrating all kinds of Grid resources, including the network resources through negotiation with local resource management systems. This kind of integrated architecture for Grid computing allows an application to request for resources in an automated manner. It brings several major advantages over a typical solution in which resources are not provisioned in an automated way, especially in the context of connectivity which is usually based on pre-established network connections between sites. In the PHOSPHORUS project, several Grid applications were adapted to validate the new PHOSPHORUS architecture [16, 17]:

- WISDOM (Wide In Silico Docking on Malaria) - a docking service which allows to compute millions of compounds of large-scale molecular dockings on 
targets implicated in diseases like malaria (in silico experimentation). WISDOM needs a arge amount of storage in order to collect output data.

- KoDaVis (Collaborative Data Visualization) - a distributed, collaborative visualization system providing remote access to a vast number of atmospheric simulation data originating from the simulation of the transport of chemical tracers in the troposphere. $\mathrm{KoDaVis}$ requires low latency and high bandwidth (approximately $700 \mathrm{Mbps}$ ).

- TOPS (Technology for Optical Pixel Streaming) - an application which enables remote viewing of large scientific datasets (2D or $3 \mathrm{D})$ on high resolution displays. TOPS requires very high bandwidth in order to transfer gigapixel scientific visualizations.

- DDSS (Distributed Data Storage Systems) - two DDSS applications were tested - the GridFTP service for transferring large amounts of data and backup/archive application used for automatic backup and archive of data from Grid nodes.

\section{A SEAMLESS MANAGEMENT OF NETWORK AND COMPUTATIONAL RESOURCES}

As stated earlier, the PHOSPHORUS automated management of both Grid and network resources enhances the efficiency of the network and Grid applications. It allows on-demand dynamic configuration of resources which results in optimized use of those resources (e.g. reduces oversubscription), significantly simplifies management and allows work in a heterogeneous, multi-domain environment.

\section{IV.1. Enhancements to the GMPLS Control Plane}

GMPLS enables automatic provisioning of connections across multiple technological domains like Ethernet, DWDM, IP, MPLS, SDH, etc. It provides uniform Control Plane solution for transport networks, facilitating integration of networks with different data plane technologies, nevertheless it can't support any Grid applications in terms of single-step, automated reservation of resources. Within the PHOSPHORUS framework enhancements to the standard GMPLS Control Plane lead to the development of Grid-enabled GMPLS ( $\mathrm{G}^{2} \mathrm{MPLS}$ ) which allows providing optical network resources as the first class Grid resources. $G^{2}$ MPLS implements the concept of Grid Network Services (GNS). GNS is a service which allows single step allocation and provisioning of both Grid and network resources. $\mathrm{G}^{2}$ MPLS is backward compatible and for a user requesting only network resources will behave just as GMPLS however, it can also provide additional features like advance reservations and support of full optical TE routing extensions [18] which might be interesting for network operators and users. To avoid the development of the application-specific architecture, the $\mathrm{G}^{2}$ MPLS development was tightly coupled with available standards to produce a generic model that can be easily used. Standards used in the definition of the $\mathrm{G}^{2}$ MPLS architecture include: ITU-T Recommendations (SG15, SG13), IETF RFCs and Internet Drafts (CCAMP WG, MPLS WG), OIF Implementation Agreements (UNI, E-NNI), OGF (ogsa-wg, jsdlwg, glue-wg, ghpn-rg).

The most important features of $\mathrm{G}^{2}$ MPLS are related to the support of enhanced network and Grid services required by the new generation of scientific applications. The Grid enabled GMPLS Control Plane controls both optical network and the Grid resources. The Grid extensions of GMPLS cover the following procedures $[19,20]$ :

- Discovery and advertisement of Grid capabilities and resources of the participating Grid sites,

- Service setup and maintenance,

- Coordination, co-allocation and configuration of Grid and network resources associated with a Grid job,

- Recovery of the installed network services and possible escalation of procedures,

- Advance reservations of Grid and network resources,

- Service monitoring: retrieving of the status of a Grid job and the related network connections.

For the $\mathrm{G}^{2}$ MPLS architecture two different Control Plane models enabling single step reservation of resources were defined: the $G^{2}$ MPLS Overlay model and the $\mathrm{G}^{2}$ MPLS Integrated model [18]. Both models treat the network in a different way, which makes the architecture more flexible.

The $\mathrm{G}^{2}$ MPLS Overlay model (Fig. 2) allows Grid middleware to take control over distributed computational resources and optical network connections. The Grid layer has all necessary information about resources and connections and coordinates all reservations. In this model the $G^{2}$ MPLS control plane is used just to create the network connections and to carry Grid information related to site capabilities and resource availabilities between the Grid sites. In case of the $\mathrm{G}^{2}$ MPLS Integrated model (Fig. 3) the network control plane is responsible for the most of coallocation functionalities. $\mathrm{G}^{2}$ MPLS configures and schedules jobs related to Grid sites as well as those related to the network. 


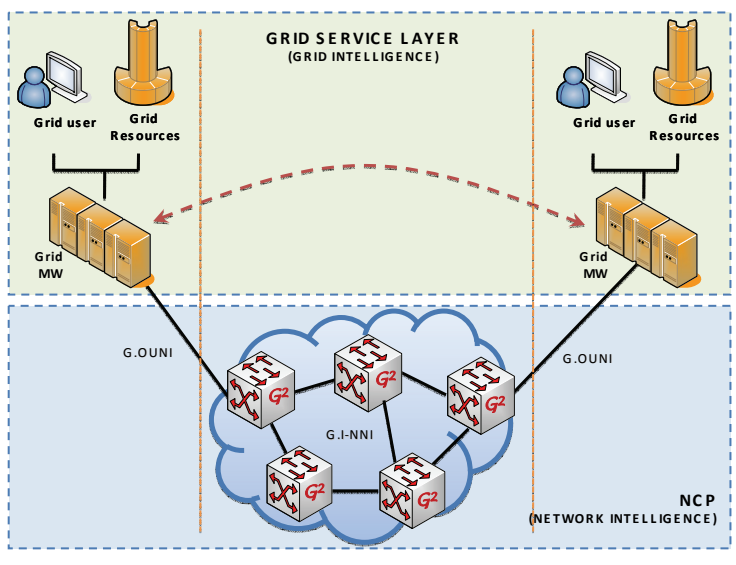

Fig. 2. $\mathrm{G}^{2}$ MPLS Overlay model [21]

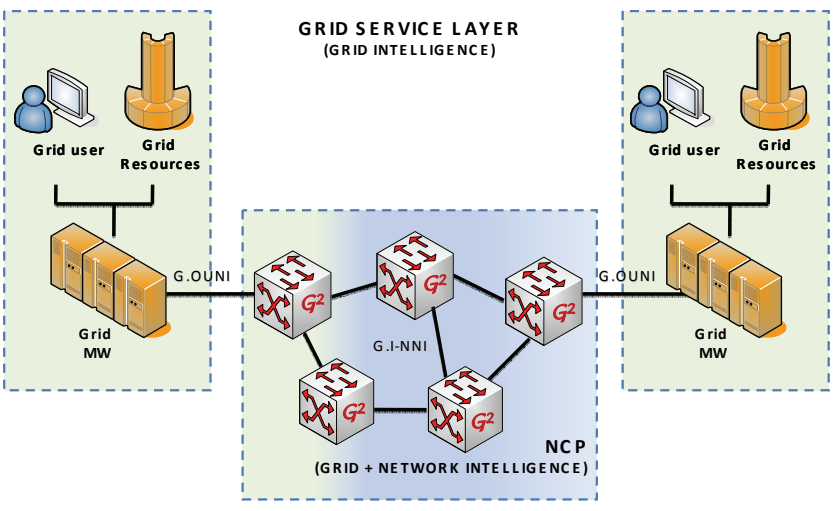

Fig. 3. $G^{2}$ MPLS Integrated model [21]

The $G^{2}$ MPLS network control plane manages Grid jobs but Grid scheduler functionality still needs to be provided by the Grid layer in order to coordinate workflow services. The two schemas that can be used for the Grid-extension of the $G^{2}$ MPLS Control Plane are: Job Submission Description Language (JSDL) [22], for the job submission and description and Grid Laboratory Uniform Environment (GLUE) schema [23] for the resources description. Additionally, special extensions to OSPF have been made in order to advertise Grid and additional network information through the network. For Grid related extensions new opaque LSA (Grid LSA) has been defined, and for network related extensions information is added to TE LSA.

To provide the required functionality, five reference points (similar to points from ASON/GMPLS architecture) have been identified [5]. These points are illustrated in Fig. 4.

- G.OUNI, i.e. the Grid Optical User-Network Interface that supports Grid and network signalling and discovery between the Grid site and the G2MPLS domain.
- G.I-NNI, i.e. the Grid Internal Node-Node Interface (G.I-NNI) that supports the routing and signaling procedures between adjacent nodes.

- G.E-NNI, i.e. the Grid External Network-Network Interface that propagates Grid and network topology information across different Control Plane domains and supports the inter-domain signaling mechanisms.

- SBI, i.e. the Southbound Interface that retrieves the resource status from the specific Transport Plane and translates Control Plane actions into appropriate configurations of those resources.

- NBI, i.e. the Northbound Interface that groups two interfaces towards upper layers: one towards the Grid layer (G.NBI) and one towards the Network Service Plane (including NRPS, N.NBI).

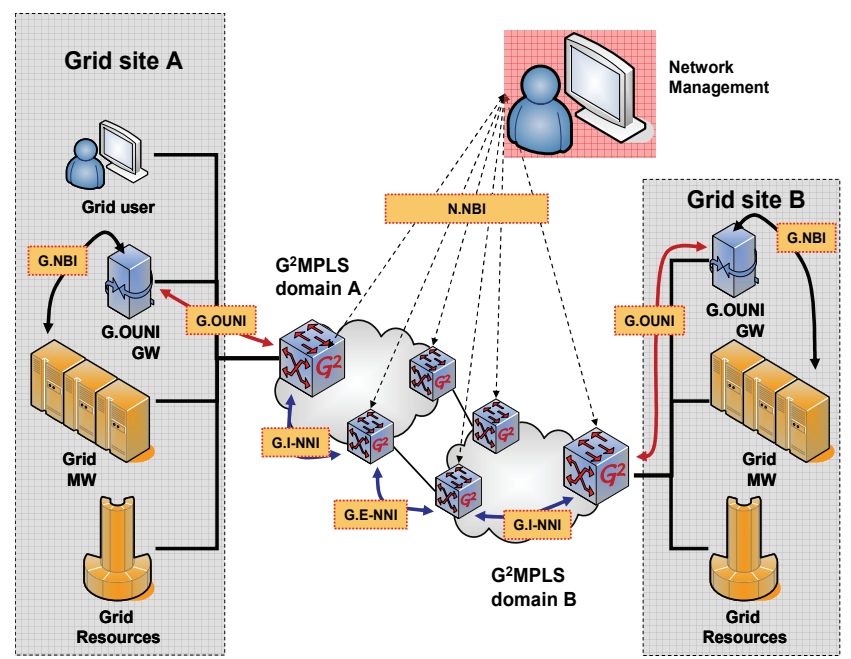

Fig. 4. $\mathrm{G}^{2}$ MPLS reference points [5]

$G^{2}$ MPLS also implements security features through support of the GAAA-TK library. This library provides all necessary functionality to support policy based on-demand network resource provisioning in a heterogeneous multidomain environment [25].

\section{IV.2. The HARMONY system}

Existing Network Resource Provisioning Systems (NRPS) can establish connections mainly in a single domain environment. The main goal of the HARMONY system was to provide interoperability between different NRPSes (ARGON, DRAC and UCLP) and GMPLS/G ${ }^{2}$ MPLS to allow end-to-end optical path reservation across different heterogeneous domains as well as provide interface which will allow scientific applications to request dynamic and auto- 


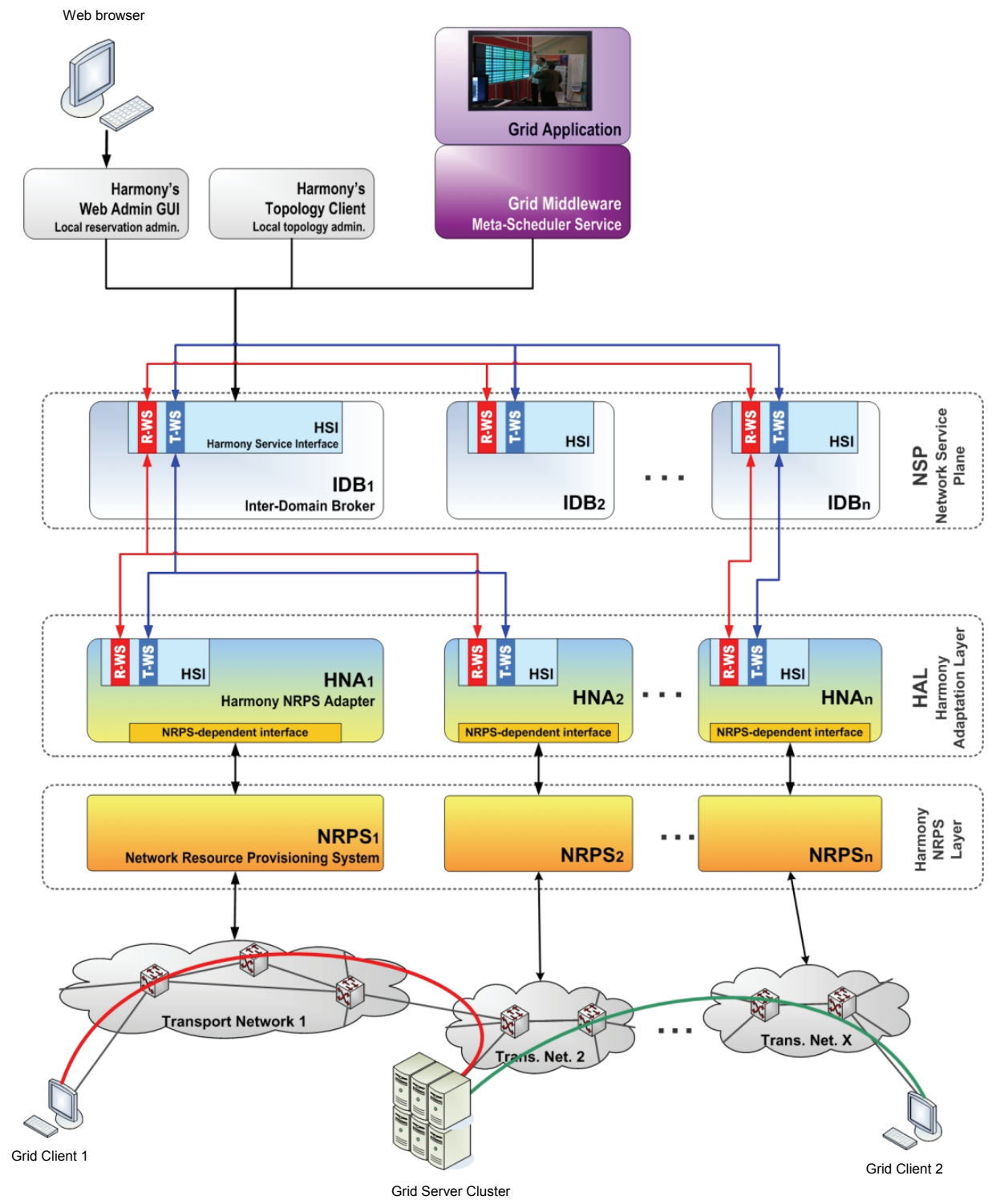

Fig. 5. HARMONY architecture [26]

mated advanced network services for Grid sites. The overall architecture of the HARMONY system is given in Fig. 5.

The most important part of the HARMONY system is the HARMONY Network Service Plane. The NSP is composed of a number of independent Inter Domain Brokers (IDBs) which control their own domains and perform topology information exchange with other IDBs. IDBs can work in different configurations allowing centralized, hierarchical and distributed model of NSP operation [2, 3]. A web service based interface, called HARMONY Interface (HSI), is used for communication with Inter Domain Brokers. The HARMONY Interface is compliant with WSRF v1.2 [29] and composed of three main components [1]:
- Reservation-WS which enables a middleware component to query resource availability, create and cancel reservations as well as query reservation status,

- Topology-WS which is used for adding, deletion and editing of domains and interdomain links as well as topology exchange between different IDBs,

- Notification-WS which is responsible for event notification management in order to implement fast responses to error situations.

Network Resource Provisioning Systems, located in the HARMONY NRPS Layer are controlled by IDBs through HARMONY NRPS Adapters (HNA) adapters to map the NRPS specific interface into a common HARMONY interface. 
HARMONY provides advanced network services to Grid middleware and end users by offering three kinds of network reservations [1]:

- Fixed reservations - in this type of reservation the user must specify start/end time of reservation and required bandwidth,

- Deferrable reservations - the user must specify the earliest start time, duration, deadline and required bandwidth,

- Malleable reservation - this type of reservation allows users to specify time frames of reservation (i.e. the earliest time when reservation could start and the latest time when the reservation could end) and minimum and maximum allowed bandwidth. A suitable slot for reservation will be found according to users' needs. This kind of reservations offers much more flexibility to the users, as the suitable time and bandwidth are proposed by HARMONY.

The following figure depicts differences between the three types of network reservations in HARMONY:

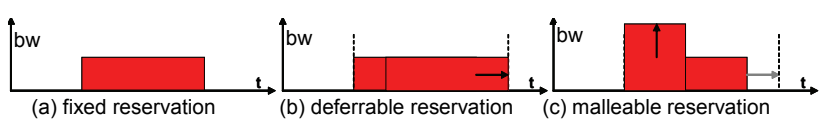

Fig. 6. Fixed, deferrable and malleable reservations in HARMONY [1]

HARMONY also supports the Authentication and Authorization Infrastructure based on the GAAA Toolkit.

\section{SUMMARY AND FURTHER WORK}

PHOSPHORUS developments successfully enabled seamless integration of optical networks and Grids. Interoperability between $\mathrm{G}^{2} \mathrm{MPLS}$ and HARMONY, as well as the compliance with Internet2 IDC and GN2 AutoBAHN have been achieved through development of proper interfaces. This enables seamless interoperation of multiple heterogeneous network domains, possibly managed by different management platforms, while cooperation with Grid middleware and applications increases efficiency of high performance computing applications. Figure 7 depicts improvements introduced by the PHOSPHORUS project to allow seamless integration of Grid and networking technologies. Grid applications (Kodavis, Wisdom, TOPS, DDSS) using PHOSPHORUS enhancements (represented by dotted lines in Fig. 7) through Grid middleware can dynamically use optical network resources to transport data between different sites.
All PHOSPHORUS developments were tested and validated in a large scale test-bed which consisted of resources scattered across Europe, North America and Korea, connected with a high speed optical network.

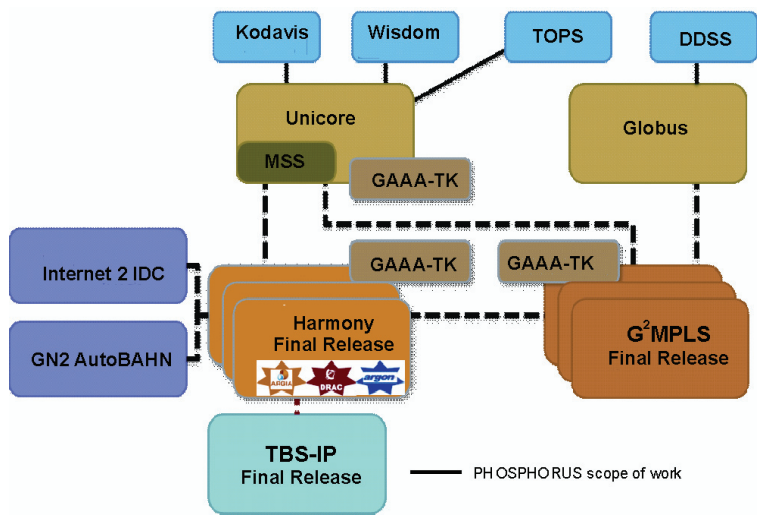

Fig. 7. PHOSPHORUS developments

The Open Source software developed in PHOSPHORUS (freely available at the PHOSPHORUS website [30]) allows further development of protocols and extensions of the network control plane. As a result, a brand new control plane, capable of carrying information not only about Grid resources, but also about any other IT resources may be potentially developed. The PHOSPHORUS ideas and vision evolved into another EU project, GEYSERS, where one of the main concepts assumes that high-end IT resources at users' premises are fully integrated with the network services procedures, both at the infrastructureplanning and connection-provisioning phases. Following this vision, GEYSERS will specify and implement a novel optical-network architecture able to support "Optical Network + Any-IT" resource provisioning seamlessly and efficiently. Energy-consumption metrics for the end-to-end service routing are part of this efficiency. These achievements will enable infrastructure providers, network operators and application providers to participate in new business scenarios where complex services with complex attributes and strict bandwidth requirements can be offered economically and efficiently to users and applications. GEYSERS's outcomes will be validated in an EU-wide optical network test-bed [31].

\section{Acknowledgments}

PHOSPHORUS was supported by the European Commission through the IP PHOSPHORUS project in the Sixth Framework Programme, contract number 034115. We would like to express the acknowledgements to all PHOSPHORUS 
partners for their great commitment on the project research and development.

\section{References}

[1] Requirements and specifications of interfaces and architecture for interoperability between NRPS, GMPLS, Middleware, PHOSPHORUS deliverable D1.1, March 2007, http://phosphorus.pl/files/deliverables/Phosphorusdeliverable-D1.1.pdf

[2] Report on Harmony System Enhancements and Testing, PHOSPHORUS deliverable D1.10, June 2009, http://www.ist-phosphorus.eu/files/deliverables/ Phosphorus-deliverable-D1.10.pdf

[3] Network Service Plane Enhancements, PHOSPHORUS deliverable D1.8, September 2008, http://www.istphosphorus.eu/files/deliverables/Phosphorus-deliverableD1.8.pdf

[4] C. Barz, M. Pilz, T. Eickermann, L. Kirtchakova, O. Waldrich, W. Ziegler: Co-Allocation of Compute and Network Resources in the VIOLA Testbed http://www.coregrid.net/mambo/images/stories/TechnicalReports/tr-0051.pdf

[5] A. Tzanakaki, G. Markidis, N. Ciulli, G. Carrozzo, G. Giorgi, E. Escalona, A. Binczewski, M. Stroiński, J. Węglarz, B. Belter, D. Parniewicz, M. Strozyk, R. Krzywania, F. Hommes, D. Simeonidou, G. Zervas, R. Nejabati, W. Doonan, Deployment Models and Solutions of the Grid-GMPLS Control Plane, PHOSPHORUS deliverable D2.6, May 2007 http://www.ist-phosphorus.eu/files/deliverables/Phosphorus-deliverable-D2.6.pdf

[6] O. Waldrich, P. Wieder, W. Ziegler, Meta-Scheduling Service for Co-allocating Arbitrary Types of Resources, CoreGRID Technical Report, Number TR-0010, December 2, 2005.

[7] http://www.nortel.com/DRAC

[8] http://dragon.maxgigapop.net/twiki/bin/view/DRAGON/ WebHome

[9] L. Battestilli, A. Hutanu, G. Karmous-Edwards, D.S. Katz, J. MacLaren, J. Mambretti, J.H. Moore, H.G. Seung-Jong Park Perros, S. Sundar, S. Tanwir, S.R. Thorpe, Yufeng Xin, EnLIGHTened Computing: An architecture for coallocating network, compute, and other grid resources for high-end applications, High Capacity Optical Networks and Enabling Technologies, 2007. HONET 2007, November 2007.

[10] http://enlightenedcomputing.org/

[11] T. Kudoh et al., G-lambda: Coordination of a Grid Scheduler and Lambda Path Service over GMPLS, Future Generation Computer Systems 22 (8), October 2006.

[12] http://www.geant2.net/server/show/nav.756

[13] http://sourceforge.net/projects/unicore/

[14] http://www.globus.org/toolkit/about.html
[15] http://www.gridge.org

[16] Report on initial adaptation of application, PHOSPHORUS Deliverable D3.3, September 2007, http://phosphorus.pl/ files/deliverables/Phosphorus-deliverable-D3.3.pdf

[17] Report on the Results of the Application Experiments During the Final Testbed Experiments, PHOSPHORUS deliverable D3.6, July 2008, http://phosphorus.pl/files/deliverables/Phosphorus-deliverable-D3.6.pdf

[18] B. Belter, G. Bernini,A. Binczewski, G. Carrozzo, N. Ciulli, E. Escalona, G. Giorgi, R. Krzywania, R. Nejabati, D. Parniewicz, F. Salvestrini, D. Simeonidou, M. Strozyk, G. Zervas, Routing and Signalling Extensions for the GridGMPLS Control Plane, PHOSPHORUS deliverable D2.2, September 2007, http://www.ist-phosphorus.eu/files/deliverables/Phosphorus-deliverable-D2.2.pdf

[19] http://www.ist-phosphorus.eu/activities.php?id=2

[20] G. Zervas, E. Escalona, R. Nejabati, D. Simeonidou, G. Carrozzo, N. Ciulli, B. Belter, A. Binczewski, M. Stroiński, A. Tzanakaki, G. Markidis, Phosphorus Grid-enabled GMPLS Control Plane (G2MPLS): Architectures, Services and Interfaces. IEEE Communications Magazine under the Special call on "Multi-Domain Optical Networks: Issues and Challenges", May 2008,

http://www.ist-phosphorus.eu/files/publications/ComMag Phosphorus v0 95.pdf

[21] B. Belter, A. Binczewski, G. Carrozzo, N. Ciulli, T. El Gorashi, J. Elmirghani, E. Escalona, G. Giorgi, R. Krzywania, G. Markidis, R. Nejabati, D. Parniewicz, F. Salvestrini, D. Simeonidou, M. Strozyk, A. Tzanakaki, O. Wäldrich, G. Zervas, Grid-GMPLS network interfaces specification, PHOSPHORUS deliverable D2.7, February 2008, http://www.ist-phosphorus.eu/files/deliverables/Phosphorus-deliverable-D2.7_M17.pdf

[22] A. Anjomshoaa et al., "Job submission description language (JSDL) specification v. 1.0", Open Grid Forum document GFD.56, November 2005, http://www.gridforum.org/documents/GFD.56.pdf

[23] S. Andreozzi (Ed.), "GLUE Schema Specification - version 1.3", Draft 3 - 16 Jan 2007, http://forge.cnaf.infn.it/plugins/scmsvn/viewcvs.php/*check out*/v_1_3/spec/pdf/GLUESchema.pdf?rev=49\&root=glue schema

[24] http://en.wikipedia.org/wiki/UNICORE

[25] http://www.ist-phosphorus.eu/software.php?id=gaaa tk

[26] http://www.ist-phosphorus.eu/activities.php?id=1

[27] http://www.viola-testbed.de

[28] http://www.itu.int/ITU-T/ngn/definition.html

[29] http://www.oasis-open.org/committees/tc_home.php?wg_abbrev=wsrf

[30] http://www.ist-phosphorus.eu

[31] http://www.geysers.eu/ 


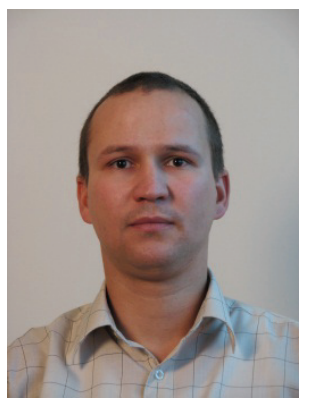

Bartosz Belter received the M.Sc. degree in Computer Science from the Poznan University of Technology in 2002. He works in Poznań Supercomputing and Networking Center as a Senior Network Engineer. He has participated in several FP6 IST projects: 6NET (IST-2001-32063), PHOSPHORUS (IST034115) and GN2 (IST511082). He has also participated in a number of national initiatives funded by the Polish Ministry of Science and Higher Education (Clusterix, Polish LDAP and others). Currently he is involved in the national project "Engineering of Future Internet" and two FP7 projects GN3 (Project No. 238875) and GEYSERS (Project No. 248657). He is a member of the TERENA Networking Conference Programme Committee. His main research activities concern the architectural aspects of Control and Management Planes in optical networks and Quality of Service in next generation packet networks.

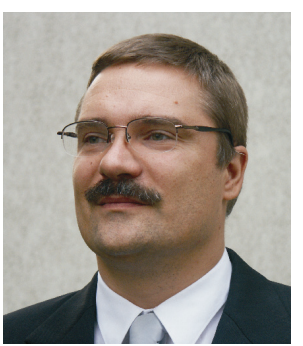

ARTUR BinczewSKI received the M.Sc. degree in Computer Science from the Poznan University of Technology in 1993. He is the Manager of Network Division at PSNC. He was involved in several EC projects: SEQUIN (IST-1999-20841), ATRIUM (IST-1999-20675), 6NET (IST-2001-32063). He coordinated the Porta Optica Study (RI026617) and PHOSPHORUS (IST034115) projects. He is author or co-author of papers in major professional journals and conference proceedings.

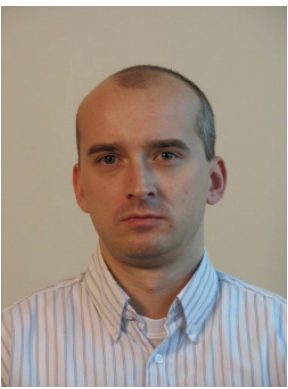

Milosz Przywecki received the M.Sc. degree in Electronics and Telecommunications from the Poznań University of Technology in 2003 and joined the Network Division of Poznan Supercomputing and Networking Center as a Networking Systems Analyst in 2005. He participated in several EC networking projects: GN2 (IST511082), Porta Optica Study (RI026617), PHOSPHORUS (IST034115). Currently he is involved in the national project "Engineering of Future Internet" and two FP7 projects GN3 (Project No. 238875) and GEYSERS (Project No. 248657). His main interests are in advanced networking technologies, network protocols and services.

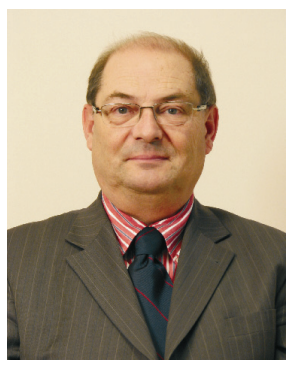

MACIEJ STRoIŃSKI is the Technical Director of Poznań Supercomputing and Networking Center, Vicepresident of the PIONIER Consortium - Polish Optical Internet and member of the Supervisory Board. He is also lecturer in the Institute of Computing Science of the Poznan University of Technology. His research interests concern networking, quality of service, network protocols and management, Grid related topics. He is author and co-author of over 150 papers in major professional journals and conference proceedings. Maciej Stroiński is the co-author of the Pionier Programme accepted by the Polish State Committee for Scientific Research and announced as the official Polish programme in 2000 . He is a member of Programme and Steering Committees of national and international conferences and editor of Computational Methods in Science and Technology journal. Maciej Stroiński has participated in several networking and Grid-related EU and national projects, cooperating with well known IT companies. He has established the Sun Center of Excellence and Microsoft Innovation Center in PSNC. 